\title{
BMJ Open Clinical effects of blood donor characteristics in transfusion recipients: protocol of a framework to study the blood donor-recipient continuum
}

Michaël Chassé, ${ }^{1}$ Lauralyn Mclntyre, ${ }^{1}$ Alan Tinmouth, ${ }^{1}$ Jason Acker,
${ }^{2}$
Shane W English, ${ }^{1,3}$ Greg Knoll, ${ }^{1}$ Alan Forster, ${ }^{4}$ Nadine Shehata, ${ }^{5}$
Kumanan Wilson, ${ }^{1,6,7}$ Carl van Walraven, ${ }^{1}$ Robin Ducharme,,${ }^{1,7}$ Dean A Fergusson ${ }^{1}$
To cite: Chassé $M$ McIntyre L, Tinmouth A, et al. Clinical effects of blood donor characteristics in transfusion recipients: protocol of a framework to study the blood donorrecipient continuum. BMJ Open 2015;5:e007412. doi:10.1136/bmjopen-2014007412

- Prepublication history for this paper is available online. To view these files please visit the journal online (http://dx.doi.org/10.1136/ bmjopen-2014-007412)

Received 10 December 2014 Accepted 22 December 2014

CrossMark

For numbered affiliations see end of article.

Correspondence to Dr Michaël Chassé; mchasse@ohri.ca

\section{ABSTRACT}

Introduction: When used appropriately, transfusion of red blood cells (RBCs) is a necessary life-saving therapy. However, RBC transfusions have been associated with negative outcomes such as infection and organ damage. Seeking explanations for the beneficial and deleterious effects of RBC transfusions is necessary to ensure the safe and optimal use of this precious resource. This study will create a framework to analyse the influence of blood donor characteristics on recipient outcomes.

Methods and analysis: We will conduct a multisite, longitudinal cohort study using blood donor data routinely collected by Canadian Blood Services, and recipient data from health administrative databases. Our project will include a thorough validation of primary data, the linkage of various databases into one large longitudinal database, an in-depth epidemiological analysis and a careful interpretation and dissemination of the results to assist the decision-making process of clinicians, researchers and policymakers in transfusion medicine. Our primary donor characteristic will be age of blood donors and our secondary donor characteristics will be donor-recipient blood group compatibility and blood donor sex. Our primary recipient outcome will be a statistically appropriate survival analysis post-RBC transfusion up to a maximum of 8 years. Our secondary recipient outcomes will include 1-year, 2-year and 5-year mortality; hospital and intensive care unit length of stay; rehospitalisation; new cancer and cancer recurrence rate; infection rate; new occurrence of myocardial infarctions and need for haemodialysis. Ethics and dissemination: Our results will help determine whether we need to tailor transfusion based on donor characteristics, and perhaps this will improve patient outcome. Our results will be customised to target the different stakeholders involved with blood transfusions and will include presentations, peer-reviewed publications and the use of the dissemination network of blood supply organisations. We obtained approval from the Research Ethics boards and privacy offices of all involved institutions.

\section{INTRODUCTION}

Transfusion of red blood cells (RBCs) is a necessary, life-saving intervention. RBCs are given to increase oxygen delivery to tissues in clinical situations where the haemoglobin level is low (anaemia). Approximately 1.1 million RBC units are collected and transfused each year in Canada. ${ }^{12}$ They are used across a variety of medical and surgical scenarios. Approximately $30 \%$ of critical care patients, and more than $50 \%$ of cardiac surgery patients will receive blood products during their hospital stay. ${ }^{3}{ }^{4}$ Owing to their common clinical use and the necessary precautions to ensure their safety, the use of blood products is associated with significant costs to the Canadian healthcare system. In 2012, the total Canadian Blood Services' (CBS) budget for transfusion products was $\$ 473$ million dollars ${ }^{1}$ and close to $\$ 366$ million dollars for Héma-Québec. ${ }^{2}$ These totals do not account for the cost of administering the blood products or the additional societal costs associated with transfusion. For RBCs, the societal cost associated with a transfusion has been estimated to be greater than $\$ 400$ per unit. $^{5}$ With more than 1.1 million RBC units delivered to Canadian hospitals and clinics in 2012, one can only begin to grasp the clinical and economic importance and impact of RBC transfusions on our healthcare system. ${ }^{1}{ }^{2}$ Given such a precious resource, we need to ensure that we maximise the benefit and minimise the risks in transfusion recipients.

Much effort has been undertaken over the past 30 years to mitigate risks and promote appropriate practice regarding the screening, collection, storage, distribution and transfusion of blood products. Indeed, the blood system has never been safer. However, there 
are potentially other factors that may contribute to transfusion safety that have not been addressed. Transfusions of RBCs are used extensively in clinical practice and the identification of novel risk factors for adverse transfusion outcomes may generate new research hypotheses to improve the safety of RBC transfusion and potentially improve clinical outcomes.

Even if anaemia is clearly associated with adverse outcomes, it is unclear if transfusion of RBCs will improve outcomes in all patients. In fact, several large and robust clinical trials $^{6-8}$ suggest that a liberal transfusion strategy (ie, transfusing blood at a higher haemoglobin concentration) is not helpful and may be harmful as compared to a restrictive transfusion strategy. Additionally, a recent systematic review suggested that RBC transfusions may be associated with increased morbidity and mortality. ${ }^{9}$ Seeking explanations for the beneficial and deleterious effects of RBC transfusions is necessary to ensure the safe and optimal use of a precious biological resource.

\section{Mitigating the risks: careful selection and screening of donors}

The risks associated with blood transfusion have been well documented and described. ${ }^{9-11}$ Critical improvements in blood compatibility testing and red cell preservation at the beginning of the 20th century allowed the large-scale use of blood for both soldiers during times of war and the general population. ${ }^{12}$ The discovery of blood-transmissible diseases such as syphilis and hepatitis $\mathrm{B}$, and the tragedies of contaminated blood with hepatitis C and HIV in the 1980s and 1990s, prompted systems changes to markedly increase the safety of blood transfusion and a keen awareness of the infectious and immunological risks associated with transfusion..$^{10}$ Owing to interventions to decrease the risk of transfusion-associated infections, the risk of transfusion-related infections is now very low (approximately 1 in 8 million for HIV, 1 in 6.7 million for hepatitis $\mathrm{C}$ and 1 in 1.7 million for hepatitis B). ${ }^{13}$

Recent evidence demonstrates that clinical outcomes following transfusion, including important mortality and mobility, are impacted by many non-infectious transfusion adverse events including acute and delayed haemolytic reactions, transfusion-related acute lung injury (TRALI), transfusion-associated circulatory overload (TACO) and hypotensive reactions. Other transfusionrelated effects such as transfusion-related immunomodulation (TRIM) are well documented in terms of their biological effects, ${ }^{10}$ but their association with clinical outcomes is not clear. 'Standard' blood products have important biological variability that can create interactions between the donor and the recipient which can impact the clinical efficacy and safety of a blood transfusion. In situations where the benefits of an intervention are uncertain, and known risks are present, it is essential that risks are minimised and potential clinical benefits are maximised. Thus, we need to examine all factors that could contribute to morbidity and mortality from the effect of RBC transfusions.
Blood donor characteristics may affect

\section{transfusion outcome}

Transfusion reactions, immune or non-immune mediated, may occur during, immediately following or be delayed in presentation after a transfusion and affect recipient outcomes. Although non-immune reactions such as citrate toxicity, air embolism and others are unlikely to be related to donor characteristics, immune reactions may be. Immune reactions in the recipient are caused by either donor cytokines (such as interleukins and tumour necrosis factor) or antibodies present in residual plasma. ${ }^{3}{ }^{10}$ Reactions may range from benign fever to very severe outcomes such as anaphylaxis, shock or death. ${ }^{14}$

Transfusion outcomes may, however, be affected by more than transfusion reactions. TRALI, an acute lung injury occurring within $6 \mathrm{~h}$ after the transfusion of a blood product, is the leading cause of transfusion-related mortality. ${ }^{15}$ Recent epidemiological studies associate the presence of human leucocytes antibodies and human neutrophil antibodies in the donors' plasma, and a history of pregnancy with the risk of TRALI (female sex, OR 4.5, 95\% CI 1.08 to 3.04$).{ }^{15}$ This led to a policy change to use male-only plasma for transfusion and a subsequent reduction in TRALI (OR 2.57, 95\% CI 1.92 to 3.86 for reduction of TRALI) in Canada. ${ }^{16}$ Similar results using TRALI reduction strategies have been observed in the $\mathrm{UK}^{17}$ and the USA. ${ }^{15}$

TACO is a common adverse complication where pulmonary oedema secondary to volume overload occurs following a blood transfusion. It is associated with increased mortality and hospital length of stay. ${ }^{18}$ It is likely that other mechanisms beyond simple fluid overload are involved since $20 \%$ of reported cases of TACO followed the transfusion of only one RBC unit. Further, fever is often associated with TACO, suggesting that there are characteristics of the blood that can contribute to events that are most likely donor related. ${ }^{19}$

Another major postulated mechanism for adverse outcomes after transfusion is TRIM. Immunomodulation secondary to transfusion has been associated with adverse clinical outcomes such as infection, acceleration of cancer growth, multiple organ dysfunction and mortality after transfusion. ${ }^{3} 20$ These transfusion-related adverse outcomes have all been suggested to be related to donor characteristics and such association needs further study.

\section{How can the donor-recipient continuum be investigated?}

Studying the impact of donor and recipient factors on outcomes following RBC transfusion is difficult due to the independent nature of the blood system (donation and preservation) and healthcare settings (crossmatching and transfusion of recipients). To perform such a study, one has to be able to track blood from 'vein-to-vein'. The blood supply chain in most parts of the world, including Canada, is not structured to facilitate such analyses. Blood is usually collected, processed, and all information pertaining to the donor is 
confidentially stored in the central database of blood service organisations. No donor information is encoded on the RBC apart from the blood group and limited information regarding the collection and manufacture process. The blood is then delivered to hospitals where it is managed by the hospital's respective blood bank. Mechanisms exist to monitor transfusion-related reactions and a 'per-case' analysis can be performed on blood units that may be associated with such reactions. If needed, the original donor may be tracked (eg, in cases of transmitted infections or TRALI) from a unique identifier on the blood unit. However, this is done manually and is labour intensive.

Haemovigilance has improved with the discovery of HIV, hepatitis B and C, and other blood transmissible pathogens. As a result, prospective registries of blood donors, tests performed on blood and the ability to trace blood have been implemented in many countries. Examples of such databases include the Serious Hazards of Transfusion (SHOT) database from the $\mathrm{UK}^{21}$ and the Retrovirus Epidemiology Donor Study (REDS) from the USA. $^{22}$ The SHOT database, which depends on outcome reporting by transfusing hospitals, records adverse reactions from blood transfusions; donor data and data for other transfused patients having no adverse event reported are not collected. The third phase of REDS will link donor and recipients to assess recipient outcomes in approximately $7-10$ years. ${ }^{23}$

Internationally, very few countries and/or organisations can follow blood from the donor to the recipient. The most advanced database of this kind is potentially the Scandinavian Donations and Transfusions (SCANDAT) database, a joint project from Sweden and Denmark that provides complete follow-up on the outcome of transfused patients. ${ }^{24}$ This group demonstrated feasibility of 'vein-to-vein' studies using this database. ${ }^{25-27}$ The Danish Transfusion Database also has the ability to track blood from donors to recipients. However, this database is less focused on transfusion recipient outcomes, and more focused on blood use. Finally, to study blood utilisation in their country, the Netherlands recently performed this type of linkage between donors and recipients, and published a study of transfusion practices. ${ }^{28}$

In Canada, the blood supply is managed in all provinces but Québec by CBS, which collects all donorrelated data. There are also several data collection strategies leading to large administrative databases within the Canadian healthcare system. Despite the potential to follow blood from donors to recipients with these vast and robust data sets, there is currently no interoperability between them. With this proposed framework, and given our information system expertise, we have the ability to create a 'vein-to-vein' infrastructure, which is key to improving the current knowledge of the clinical impact of RBC transfusion. With our unique position, we aim to create a framework that will allow the investigation of the effect of blood donor characteristics on outcomes of transfusion recipients.

\section{METHODS AND ANALYSIS}

We will conduct a multisite, retrospective, longitudinal cohort study using data collected from blood donors by CBS, and clinical short-term and long-term outcome data from hospital and provincial health administrative databases. This study will include the thorough validation of the primary data if needed, the linkage of the various databases into one large longitudinal database, an in-depth epidemiological analysis to answer our questions, and a careful interpretation and dissemination of the results to assist the decision-making process of clinicians, researchers and policymakers in transfusion medicine. Our study will serve as a framework to develop future blood transfusion surveillance programmes to allow for the comprehensive monitoring of the blood donor-recipient continuum.

\section{Research question}

The target population will be all patients who receive an RBC transfusion. The studied exposures include donor age as the main exposure, and donor sex, donor-recipient compatibility and blood type as secondary exposures. Our primary outcome will be a statistically appropriate recipient survival analysis post-RBC transfusion up to a maximum of 8 years. Our secondary outcomes will include 1-year, 2-year and 5-year mortality; hospital and intensive care mortality; hospital and intensive care unit length of stay; rehospitalisation; new cancer and cancer recurrence rate; infection rate (methicillin-resistant Staphylococcus aureus and Clostridium difficile as validated infectious outcomes and surrogates for hospital-acquired infections); new occurrence of myocardial infarctions and the need for haemodialysis (as a surrogate for severe chronic renal failure). These secondary outcomes were selected both based on the quality and accuracy of these outcomes in the source registries, and in order to cover a clinically representative range of adverse short-term and long-term events after transfusion (mortality, cardiovascular, oncology, mortality, infections, renal). The planned study time frame will be from 25 October 2006 to 31 December 2013. At this stage of the programme, we will include the following hospitals in the Ottawa region: The Ottawa HospitalGeneral Campus, The Ottawa Hospital-Civic Campus, The University of Ottawa Heart Institute and The Ottawa Hospital—Riverside Campus.

\section{Source of data}

We will obtain the data for the required analyses from different sources. Recipient data will first be obtained from The Ottawa Hospital (TOH) Data Warehouse. TOH Data Warehouse integrates data from several systems used at the hospital including, but not limited to, patients, encounters, services, emergency visits, census information, health records abstracts, facility and capacity history and laboratory information services. The data are entered in their respective systems and then transformed and reformatted to be stored centrally at $\mathrm{TOH}$. Additional outcome data will be obtained from the 
Institute for Clinical Evaluative Sciences (ICES). ICES houses Ontario's health administrative databases. The most relevant ICES data sets for this study include the Canadian Institute for Health Information's Discharge Abstract Database, the Ontario Cancer Registry, the Ontario Health Insurance Plan database, the Registered Persons Database and the Ontario Drug Benefit database. ${ }^{29}$ Data linkage at ICES will allow us to measure patient survival beyond the initial hospitalisation, and to collect information on further hospitalisations, renal and cardiovascular outcomes, as well as cancer-related information.

Donor information will be obtained from the CBS database. The CBS database includes demographic information on blood donors, the units of all collected blood and the results of the biological tests performed on the individual blood donations. The objective of this database is to collect basic health information, high-risk activities and blood characteristics on blood donors, such as $\mathrm{ABO}$ group and microbiological testing. This information is then used to exclude high-risk donors before or after they give blood for safety of the donor or the recipients, and serves as a repository of information for trace-back investigations of any adverse transfusion events. ${ }^{30}$

\section{Identification of transfused patients}

We will include any patient, hospitalised or not, who received one or more allogeneic RBC units between 25 October 2006 and 31 December 2013. The 25 October 2006 was the date when all blood products transfused started to be systematically stored centrally in the different included institutions. We will exclude patients who received autologous, directed or dedicated RBC transfusions.

\section{Identification of blood donors}

The donors will be identified from the unique RBC transfusion unit numbers from the units given to the recipient. We will not have any a priori exclusion criteria for the identification of donors.

\section{Database creation and linkage strategy}

We will first identify all patients over the 8-year period who received at least one allogeneic RBC unit. Patients who received blood up to 31 December 2013 will be included in the study. For each included patient, we will collect the age, sex, hospital service where the transfusion occurred, primary diagnosis, $\mathrm{ABO}$ and $\mathrm{Rh}$ blood type, comorbid illnesses (using the validated Charlson index), ${ }^{31-33}$ and pretransfusion haemoglobin values. Short-term outcomes (hospital mortality, hospital and intensive care length of stays) will also be gathered from TOH Data Warehouse (figure 1).

Using the unique RBC unit number of each blood product transfused, we will perform a direct linkage with the CBS database to the corresponding blood donor and perform appropriate linkage and data harmonisation for analysis (figure 2). For long-term outcomes (mortality, rehospitalisation events, terminal renal

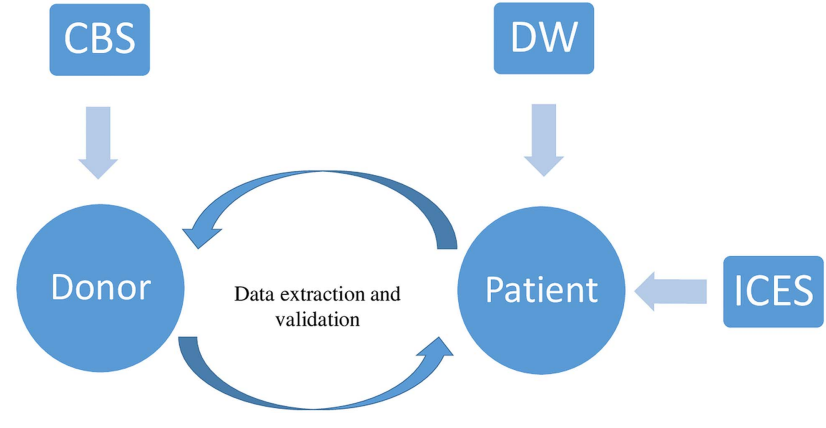

Figure 1 Schematisation of the framework. ICES, Institute for Clinical Evaluative Sciences; CBS, Canadian Blood Services; DW, Data Warehouse.

failure, myocardial infarctions and cancer rates), we will use the patient's Ontario Health Insurance Plan number to link information from $\mathrm{TOH}$ Data Warehouse to patient data housed at ICES.

\section{Sample size}

From preliminary descriptive work performed at the included centres, we estimate that more than 200000 RBC transfusions occurred during the study period, representing approximatively 35000 unique blood recipients. The number of blood donors associated with these patients cannot be estimated at this time.

\section{Data quality and validation}

All blood donors in Canada except for the Province of Québec are required to register with CBS before donating blood. Therefore, the registry of blood donors is complete. Data regarding the blood donor are selfreported. CBS performs validation procedures to make sure that the information reported by the donor is encoded properly in the database. The same is true for the results of the tests performed on the blood units. Therefore, the information needed on the donor (unique identifier, age, sex, $\mathrm{ABO}$ and $\mathrm{Rh}$ group) is accurate and does not need validation for this project.

Data quality and accuracy for recipients will depend on the studied variable or outcome. Objective information such as age, sex and $\mathrm{ABO}$ and $\mathrm{Rh}$ group are expected to be valid as they are subject to internal validation. Age and sex are entered automatically on scanning of the patient's healthcare number, and the $\mathrm{ABO}$ group is transferred directly from the laboratory information system. TOH Data Warehouse contains clinical data regarding comorbidities using ICD-10CA codes for coding, as well as administrative information. The validity of these codes for exposure and outcome may vary from one variable to another and incorrectly assuming the validity of these codes may lead to biased results. ${ }^{34} 35$ To address possible bias, the validity of each variable will be assessed when needed. For codes and outcomes for which the validity is not known, random manual chart 


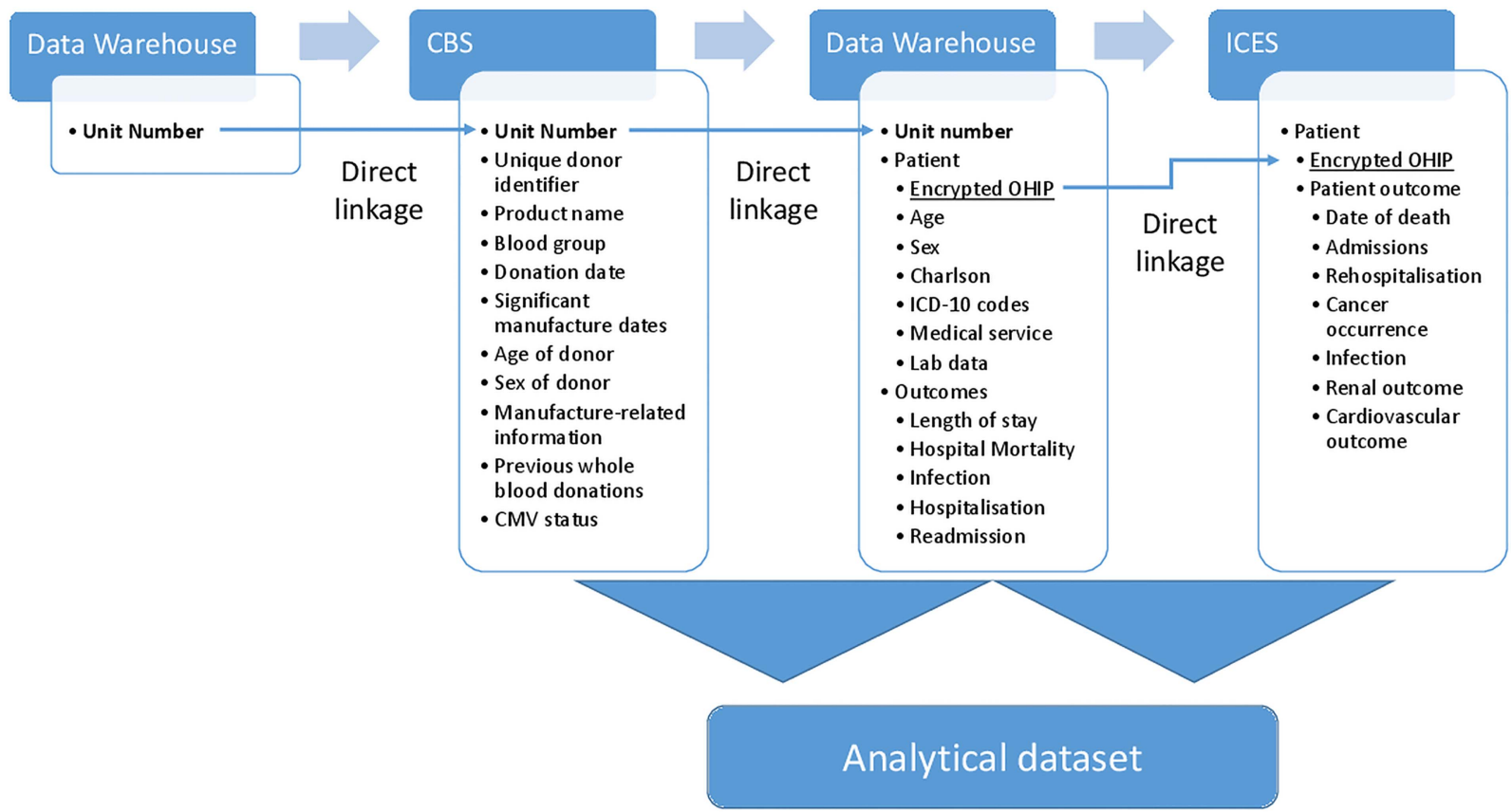

Figure 2 Data sources and linkage strategy. ICES, Institute for Clinical Evaluative Sciences; CBS, Canadian Blood Services; CMV, cytomegalovirus; ICD-10, International Classification of Diseases version 10; OHIP, Ontario Health Insurance Plan.

abstraction will be performed to compute the sensitivity and specificity of the code.

\section{Primary analysis}

Our primary analysis will aim to investigate the association between donor age and recipient outcome. The most important analytical issue for our primary analysis is that of modelling multiple transfusions of varying transfusion donor ages. ${ }^{36}$ Issues of analysis are difficult and none are ideal (mean donor age, median donor age, oldest donor age unit received, youngest donor age unit received, etc). We have considered the varying age of donors, and we will conduct an adjusted analysis by entering the donor age of each unit received as a continuous variable. In order to evaluate the effect of donor age on mortality in transfused recipients, we will use clustered Cox regression analysis with time-dependent stratification, an approach used with success in prior similar studies. ${ }^{35} 3738$ Time-dependent strata will be defined by the cumulative number of transfusions received over time. The time-dependent stratification allows us to account for mortality risk at each RBC transfusion until the end of follow-up (maximum 8 years) or death. Observations will be considered as censored for outcomes that did not occur by the end of follow-up (31 December 2013). We will adjust for important risk factors and potential confounders including age of the recipient, sex, $\mathrm{ABO}$ and $\mathrm{Rh}$ blood type, donor-recipient $\mathrm{ABO}$ match, primary diagnosis, comorbid illnesses (using the Charlson index), storage and manufacturing characteristics. Continuous risk factors will be entered into the models as continuous rather than categorical measures to improve statistical efficiency. For time- varying covariates, time will be measured from the first transfusion. Regression diagnostics will be performed on all models.

\section{Sensitivity analysis to assess the robustness of our primary analysis}

While our primary analysis will consider donor age as a continuous variable, we will also assess the cumulative exposure to older donor blood and potential dose effects by categorising donor age of transfused products using quartiles of the overall donor age distribution as discrete cut points as well as categorising donor age in discrete categories $(<18,<45,45-60,>61)$. By treating donor age as categorical variables in the past three approaches, it will be possible to investigate doseresponse relationships between age of blood donor and risk of death. We will also derive a population of patients that received only one blood transfusion over the time frame of the study, hence obtaining a 'pure' cohort of patients exposed to a given characteristic.

\section{Planned secondary analyses}

As per our primary analysis, we will implement Cox proportional hazard models with time-dependent analysis to assess the effects of donor $\mathrm{ABO}$ and $\mathrm{Rh}$ blood types, donor-recipient compatibility and sex on the risk of mortality. New cancer rates, cancer recurrence rates, $C$. difficile and methicillin-resistant $S$. aureus, terminal renal failure and myocardial infarction will be considered as categorical or counts and a similar analytical strategy as our primary outcome will be used. This method has been used before for similar exposures and outcomes. ${ }^{39}$ Hospital and intensive care unit length of stay and 
rehospitalisation rates will be analysed only for patients who were hospitalised and transfused and measured as 'time-to-discharge' or 'time-to-event'. Therefore, Cox proportional hazard models will also be appropriate.

\section{Planned subgroup analyses}

Secondary analyses are planned for the following subgroups of patients: (1) $1 \mathrm{vs} \geq 2 \mathrm{RBC}$ transfusion episodes while in hospital; (2) patients who received multiple transfusions from the same donor compared with those who received transfusions from multiple donors with comparable characteristics; (3) patient type (medical, surgical, trauma); (4) severity of illness at baseline, as measured by the Charlson index; (5) interaction between study exposures (age X sex, age X blood group) to investigate the presence of effect modification. The interaction analysis is important because patients of similar age may receive blood from different sexes, blood groups or other characteristics. A significant interaction would suggest a different impact of age depending on the other characteristics of the donor. The analytical approach described above for both primary and secondary outcome measures will be undertaken in all subgroup analyses. These analyses will primarily be hypothesisgenerating and hypothesis-supporting in nature.

\section{Risk of bias and confounding}

Epidemiological studies are subject to selection and information biases. For this proposal, the risk of selection bias is limited since we will include the entire population of patients transfused during the study time frame and we will use a complete registry that encompasses all blood donors. Regarding information bias, the studied exposures such as age, sex, blood groups and blood mismatch are objective and accurately encoded in validated registries.

To obtain valid estimates of association between an exposure and an outcome, the usual strategy in cohort studies is to adjust for known confounders. Unknown confounders are then unaccounted for and can be distributed unevenly between exposed and non-exposed groups, confounding the effect estimates. Randomisation is used to evenly distribute these unmeasured confounders between groups and reduce their impact on the effect estimation, moving observed effects towards the null hypothesis. By the nature of transfusion exposure, the unmeasured confounders will also tend to be evenly distributed between groups. Donor characteristics are always strictly concealed from any caregiver in the hospital where the blood units are used and blood is distributed in a random manner across hospitals. Therefore, the exposure of interest is randomly distributed among recipients. Owing to this concealment of the identity and characteristics of the donors, our study will also have similarities with features usually seen in RCTs which protect from sources of bias, such as allocation concealment (allocation of the donors' characteristics is concealed from any health personnel involved in the transfusion of the blood product) and double-blinding (neither the clinicians nor the patients know the donors' characteristics). ${ }^{40}$ We will carefully document potential confounders on the donor side (sex, blood group, blood characteristics, preservatives, different manufacture processes) and on the recipient side (age, comorbidities, severity of illness using the Charlson index) and adjust for them if appropriate.

Use of inclusive and valid databases from ICES and TOH Data Warehouse will also limit the number of missing values. Although we expect missing values to be minimal, we will report them and perform appropriate multiple imputation and censoring if we find missing covariates and outcome values.

\section{Adjustment and stratification for manufacture characteristics}

The blood product preparation process following donation of RBCs may affect transfusion outcomes and involves a number of manufacturing steps which may affect transfusion outcomes including the anticoagulant solution, preservative and potentially the duration of storage. At the time of donation, whole blood is collected into a plastic bag with an anticoagulant solution (citrate, phosphate and dextrose). The whole blood is then centrifuged and the RBCs are separated from other blood constituents and filtered to reduce the amount of leucocytes (leucoreduction). In Canada, SAGM (saline, adenine, glucose and mannitol) is added to optimise red cell storage and survival. Once processed, the RBCs are stored in sterile bags for up to 42 days at a temperature between $1^{\circ} \mathrm{C}$ and $6^{\circ} \mathrm{C} .{ }^{42}$ Any step of this process has the potential to affect the transfusion outcome. RBC storage solutions, such as AS-3 (citric acid, phosphate, chlorine, adenine, dextrose, citrate) and SAGM, have been demonstrated to increase the survival of red cells after transfusion. The AS-3 solution has been shown to increase storage time and to provide a better quality of RBCs. ${ }^{43}$ Regarding storage time, retrospective studies, prospective non-randomised studies, systematic reviews and meta-analysis reached conflicting results regarding the impact of storage time of RBC on recipients' outcome. ${ }^{44-}$ 47 Our randomised clinical trial in a vulnerable and heavily transfused population of neonates found no clinical benefit of fresh RBCs. ${ }^{48}$ Prospective randomised trials are underway to try and answer this question. ${ }^{49}$ While awaiting the results of these important clinical studies, this project will have to take into consideration the potential impact of these potential confounders by describing their distribution in the transfused population, adjusting or stratifying when appropriate.

\section{ETHICS AND DISSEMINATION}

\section{Ethics and privacy}

Owing to the nature of the data used for this project, individual patient consent will be waived, in accordance with the Ontario Personal Health Information Protection Act. We obtained approval from the Research Ethics 
boards of all involved institutions, as well as from privacy offices at CBS, ICES and TOH Data Warehouse. All data collection and management will be performed in accordance with the Personal Health Information Protection Act of Ontario, Regulation 329/04. All patients will be identified by a unique anonymous number and no patient identifiers will be stored with clinical data. The resulting database will be encrypted and stored centrally. Access will be restricted and only designated members of the study team will have access to the data set.

\section{Knowledge transfer plan}

The results of our study will help determine whether we need to tailor blood based on donor characteristics, and perhaps this will improve utilisation of blood and, more importantly, improve patient outcomes. Our study will have a significant impact on the way we evaluate blood transfusions. Having the capacity to rigorously study blood from 'vein-to-vein' will allow the pursuit of many new hypotheses regarding the use of blood products. An example of application of our study results would be to confirm the current age policy in blood donation. On the other hand, different outcomes according to age would support further study. For example, adverse outcomes associated with blood donated by older donors may only result in adverse outcomes for specific recipient populations, such as patients with significant comorbidities. This new information will inform policymakers and transfusion organisations regarding current policies either to support them or to use this new information as a foundation for further research in the field of transfusion medicine. It may lead to the development of validation or prospective studies regarding factors that may affect outcomes of RBC transfusion.

From the early development of this research project, we involved stakeholders and experts in a wide range of fields involved with the organisation, research and the care of patients receiving transfusions (haematologists, intensivists, transfusion specialists, transplantation specialists, healthcare researchers, epidemiologists, blood organisation decision makers and senior scientists). This diversity of expertise will ensure that the research questions, objectives, methods and result analysis and interpretation answer pertinent questions not only for clinicians, but also for stakeholders, patients and the overall population. Our design will allow us to perform the required analyses to impact on the major blood users. The results will also inform blood supply organisations policies.

Results obtained from this research project will be customised to target the different stakeholders involved with blood transfusions. For clinicians and researchers, traditional dissemination will be used, including publication in relevant peer-reviewed medical journals and presentations at local, national and international conferences and meetings. We will also use the dissemination network of CBS, for example, by presenting the results on their website for the general public. We will ensure that publications resulting from this work are open access. We will work closely with the different stakeholders of CBS and the different clinical specialties to provide reports for the specific needs and realities of their organisations/disciplines. Many additional stakeholders such as other medical specialty organisations involved in transfusion, and other blood supply organisations such as the Red Cross in the USA may benefit from the results of this study. In addition to traditional dissemination strategies, we plan to directly reach out to these organisations to present our results, and the team members will be readily available for further discussions, meetings or presentations to answer their specific needs and questions.

Through our study, we will develop an analytical framework that will enable the study of factors that may affect outcomes related to blood transfusion. We believe that our study will shed light on important hypotheses such as the influence of blood donor age and sex, and potential other characteristics in transfusion recipients, and will allow to support (or not) current policies, and potentially suggest new research ideas to improve the safety of our blood system. Such findings will have implications for the blood and healthcare systems, donors and clinicians.

\section{Author affiliations}

${ }^{1}$ Clinical Epidemiology Program, Ottawa Hospital Research Institute, Ottawa, Ontario, Canada

${ }^{2}$ Centre for Innovation, Canadian Blood Services, Edmonton, Alberta, Canada ${ }^{3}$ Department of Medicine (Critical Care), University of Ottawa, Ottawa, Ontario, Canada

${ }^{4}$ Ottawa Hospital, Ottawa, Ontario, Canada

${ }^{5}$ Department of Medicine and Laboratory Medicine and Pathobiology, University of Toronto, Mount Sinai Hospital, Toronto, Ontario, Canada ${ }^{6}$ Department of Medicine, University of Ottawa, Ottawa, Ontario, Canada ${ }^{7}$ Institute for Clinical Evaluative Sciences, Ottawa, Ontario, Canada

Contributors All authors participated in conceiving this study. MC, DAF, AT, JA, SWE, LM, KW, GK, AF, KW and NS secured its funding. MC, AT, JA, RD and DAF obtained ethics and privacy approval. MC and DAF developed the analytical plan. MC drafted the manuscript. All authors provided input into the protocol, critical feedback on the manuscript and approved the final manuscript.

Funding This work was supported by a Canadian Blood Services and Canadian Institute of Health Research Partnership on Blood Supply Risk, grant number CIHR-BSR201403-DF-326493. JA is a Scientist at Canadian Blood Services and participated in conceiving this study.

Competing interests None.

Ethics approval The Ottawa Hospital Research Ethics Board and Canadian Blood Services Research Ethics Board.

Provenance and peer review Not commissioned; peer reviewed for ethical and funding approval prior to submission.

Open Access This is an Open Access article distributed in accordance with the Creative Commons Attribution Non Commercial (CC BY-NC 4.0) license, which permits others to distribute, remix, adapt, build upon this work noncommercially, and license their derivative works on different terms, provided the original work is properly cited and the use is non-commercial. See: http:// creativecommons.org/licenses/by-nc/4.0/

\section{REFERENCES}

1. Canadian Blood Services 2012 Annual Report. Ottawa. 2012.

2. Rapport annuel 2012-2013 d'Héma-Québec. Québec. 2012.

3. Raghavan M, Marik PE. Anemia, allogenic blood transfusion, and immunomodulation in the critically ill. Chest 2005;127:295-307. 
4. Bennett-Guerrero $\mathrm{E}$, Zhao $\mathrm{Y}$, O'Brien SM, et al. Variation in use of blood transfusion in coronary artery bypass graft surgery. JAMA 2010;304:1568-75.

5. Amin M, Fergusson D, Wilson $\mathrm{K}$, et al. The societal unit cost of allogenic red blood cells and red blood cell transfusion in Canada. Transfusion 2004;44:1479-86.

6. Hébert PC, Wells G, Blajchman MA, et al. A multicenter, randomized, controlled clinical trial of transfusion requirements in critical care. Transfusion Requirements in Critical Care Investigators, Canadian Critical Care Trials Group. N Engl J Med 1999;340:409-17.

7. Hajjar LA, Vincent J-L, Galas FRBG, et al. Transfusion requirements after cardiac surgery: the TRACS randomized controlled trial. JAMA 2010;304:1559-67.

8. Carson JL, Terrin ML, Noveck $\mathrm{H}$, et al. Liberal or restrictive transfusion in high-risk patients after hip surgery. N Engl $\mathrm{J}$ Med 2011;365:2453-62.

9. Marik PE, Corwin HL. Efficacy of red blood cell transfusion in the critically ill: a systematic review of the literature. Crit Care Med 2008;36:2667-74.

10. Gilliss BM, Looney MR, Gropper MA. Reducing noninfectious risks of blood transfusion. Anesthesiology 2011;115:635-49.

11. Vamvakas EC, Blajchman MA. Transfusion-related mortality: the ongoing risks of allogeneic blood transfusion and the available strategies for their prevention. Blood 2009;113:3406-17.

12. History of Blood Transfusion. Am Red Cross 2013. http://www. redcrossblood.org/learn-about-blood/history-blood-transfusion

13. O'Brien SF, Yi Q-L, Fan W, et al. Current incidence and residual risk of HIV, HBV and HCV at Canadian Blood Services. Vox Sang 2012;103:83-6.

14. Jacobs JFM, Baumert JL, Brons PP, et al. Anaphylaxis from passive transfer of peanut allergen in a blood product. $N$ Engl $J$ Med 2011;364:1981-2

15. Toy P, Gajic O, Bacchetti P, et al. Transfusion-related acute lung injury: incidence and risk factors. Blood 2012;119:1757-67.

16. Lin Y, Saw C-L, Hannach B, et al. Transfusion-related acute lung injury prevention measures and their impact at Canadian Blood Services. Transfusion 2012;52:567-74.

17. Chapman CE, Williamson LM. National Blood Service TRAL Reduction Policies: implementation and effect. Transfus Med Hemother 2008;35:93-6.

18. Murphy EL, Kwaan N, Looney MR, et al. Risk factors and outcomes in transfusion-associated circulatory overload. $\mathrm{Am} \mathrm{J} \mathrm{Med}$ 2013;126:357.e29-38.

19. Andrzejewski C, Casey MA, Popovsky MA. How we view and approach transfusion-associated circulatory overload: pathogenesis, diagnosis, management, mitigation, and prevention. Transfusion 2013:53:3037-47.

20. Lannan KL, Sahler J, Spinelli SL, et al. Transfusion immunomodulation- the case for leukoreduced and (perhaps) washed transfusions. Blood Cells Mol Dis 2013;50:61-8.

21. Stainsby $D$, Jones $H$, Asher $D$, et al. Serious hazards of transfusion: a decade of hemovigilance in the UK. Transfus Med Rev 2006;20:273-82.

22. Schreiber GB, Busch MP, Kleinman SH, et al. The risk of transfusion-transmitted viral infections. The Retrovirus Epidemiology Donor Study. N Engl J Med 1996;334:1685-90.

23. Recipient Epidemiology and Donor Evaluation Study-III. Natl Heart Lung Blood Inst 2013. https://reds-iii.rti.org/ (accessed 9 Sep 2013)

24. Edgren G, Hjalgrim $\mathrm{H}$. Epidemiological considerations for the use of databases in transfusion research: a Scandinavian perspective. Curr Opin Hematol 2010;17:596-601.

25. Edgren G, Hjalgrim H, Reilly M, et al. Risk of cancer after blood transfusion from donors with subclinical cancer: a retrospective cohort study. Lancet 2007;369:1724-30.

26. Edgren G, Kamper-Jørgensen M, Eloranta S, et al. Duration of red blood cell storage and survival of transfused patients (CME). Transfusion 2010;50:1185-95.
27. Edgren $\mathrm{G}$, Hjalgrim $\mathrm{H}$, Rostgaard $\mathrm{K}$, et al. Risk of gastric cancer and peptic ulcers in relation to $\mathrm{ABO}$ blood type: a cohort study. $\mathrm{Am} J$ Epidemiol 2010;172:1280-5.

28. Borkent-Raven BA, Janssen MP, van der Poel CL, et al. The PROTON study: profiles of blood product transfusion recipients in the Netherlands. Vox Sang 2010;99:54-64.

29. Mission and Goals. Inst Clin Eval Sci. http://www.ices.on.ca/ (accessed 20 May 2013).

30. Donor Questionnaire. Can Blood Serv. http://www.blood.ca/ (accessed 20 May 2013).

31. Needham DM, Scales DC, Laupacis A, et al. A systematic review of the Charlson comorbidity index using Canadian administrative databases: a perspective on risk adjustment in critical care research. $J$ Crit Care 2005;20:12-19.

32. Sundararajan V, Henderson T, Perry C, et al. New ICD-10 version of the Charlson comorbidity index predicted in-hospital mortality. J Clin Epidemiol 2004;57:1288-94.

33. Sharabiani MTA, Aylin P, Bottle A. Systematic review of comorbidity indices for administrative data. Med Care 2012;50:1109-18

34. Van Walraven C, Bennett C, Forster AJ. Administrative database research infrequently used validated diagnostic or procedural codes. $J$ Clin Epidemiol 2011;64:1054-9.

35. Van Walraven C, Austin P. Administrative database research has unique characteristics that can risk biased results. $J$ Clin Epidemiol 2012:65:126-31.

36. Middelburg RA, le Cessie S, Briët E, et al. A solution to the problem of studying blood donor-related risk factors when patients have received multiple transfusions. Transfusion 2010:50:1959-66.

37. Eikelboom JW, Cook RJ, Liu Y, et al. Duration of red cell storage before transfusion and in-hospital mortality. Am Heart $J$ 2010;159:737-43.e1.

38. Wong J, Taljaard M, Forster AJ, et al. Addition of time-dependent covariates to a survival model significantly improved predictions for daily risk of hospital death. J Eval Clin Pract 2013;19:351-7.

39. Forster AJ, Taljaard M, Oake N, et al. The effect of hospital-acquired infection with Clostridium difficile on length of stay in hospital. CMAJ 2012;184:37-42.

40. Moher D, Pham B, Jones a, et al. Does quality of reports of randomised trials affect estimates of intervention efficacy reported in meta-analyses? Lancet 1998;352:609-13

41. Wood L, Egger M, Gluud LL, et al. Empirical evidence of bias in treatment effect estimates in controlled trials with different interventions and outcomes: meta-epidemiological study. BMJ 2008;336:601-5.

42. Gwen C, Blajchman M. Clinical Guide to Transfusion. Toronto, Canada: Canadian Blood Services, 2007:1-182.

43. Hess JR, Greenwalt TG. Storage of red blood cells: new approaches. Transfus Med Rev 2002;16:283-95.

44. Lelubre C, Piagnerelli M, Vincent J-L. Association between duration of storage of transfused red blood cells and morbidity and mortality in adult patients: myth or reality? Transfusion 2009;49:1384-94

45. Tinmouth A, Fergusson D, Yee IC, et al. Clinical consequences of red cell storage in the critically ill. Transfusion 2006;46: 2014-27.

46. Vamvakas EC. Meta-analysis of clinical studies of the purported deleterious effects of "old" (versus "fresh") red blood cells: are we at equipoise? Transfusion 2010;50:600-10.

47. Triulzi DJ, Yazer MH. Clinical studies of the effect of blood storage on patient outcomes. Transfus Apher Sci 2010;43:95-106.

48. Fergusson DA, Hébert $\mathrm{P}$, Hogan DL, et al. Effect of fresh red blood cell transfusions on clinical outcomes in premature, very low-birth-weight infants: the ARIPI randomized trial. JAMA 2012;308:1443-51.

49. Aubron C, Nichol A, Cooper DJ, et al. Age of red blood cells and transfusion in critically ill patients. Ann Intensive Care 2013;3:2. 